Article type : Research Papers

\title{
Secondary contact zones of closely-related Erebia butterflies overlap with narrow phenotypic and parasitic clines
}

\author{
Kay Lucek ${ }^{1 \#}$, Roger Butlin ${ }^{2,3^{*}}$, Theofania Patsiou ${ }^{1 *}$ \\ ${ }^{1}$ Department of Environmental Sciences, University of Basel, Schönbeinstrasse 6, CH- \\ 4056 Basel, Switzerland \\ ${ }^{2}$ Department of Animal and Plant Sciences, University of Sheffield, UK \\ ${ }^{3}$ Department of Marine Sciences, Tjärnö, University of Gothenburg, 45296 Strömstad, \\ Sweden \\ * in alphabetical order \\ \# Phone/Fax: +4161 2072314
}

Corresponding author email id: kay.lucek@unibas.ch

This article has been accepted for publication and undergone full peer review but has not been through the copyediting, typesetting, pagination and proofreading process, which may lead to differences between this version and the Version of Record. Please cite this article as doi: $\underline{10.1111 / J E B .13669}$

This article is protected by copyright. All rights reserved 


\begin{abstract}
Zones of secondary contact between closely related taxa are a common legacy of the Quaternary ice ages. Despite their abundance, the factors that keep species apart and prevent hybridisation are often unknown. Here we study a very narrow contact zone between three closely related butterfly species of the Erebia tyndarus species complex. Using genomic data, we first determined if gene flow occurs and then assessed whether it might be hampered by differences in chromosome number between some species. We found interspecific gene flow between sibling species that differ in karyotype by one chromosome. Conversely, only F1 hybrids occurred between two species that have the same karyotype, forming a steep genomic cline. In a second step, we fitted clines to phenotypic, ecological and parasitic data to identify the factors associated with the genetic cline. We found clines for phenotypic data and the prevalence of the endosymbiont parasite Wolbachia to overlap with the genetic cline, suggesting that they might be drivers for separating the two species. Overall our results highlight that some gene flow is possible between closely-related species despite different chromosome numbers, but that other barriers restrict such gene flow.
\end{abstract}

Key words: RADseq, Wolbachia, speciation, butterfly, clines 


\section{Introduction}

During the Quaternary ice ages, the distribution ranges of many species became fragmented, and populations subsequently diverged from each other in allopatry. Following the glacial retreats, many of these isolated populations underwent range expansions, often leading to secondary contact zones between closely-related lineages or species (Hewitt, 2000). The evolutionary outcomes of such secondary contacts depend on the presence and strengths of pre- or postzygotic intrinsic and/or extrinsic barriers to gene flow (Marie Curie SPECIATION Network et al., 2012; Seehausen et al., 2014; Canestrelli et al., 2016). The results can range from the extensive admixture of two lineages to rare hybridisation events, resulting in hybrid zones that are more or less wide depending on the degree of divergent selection or selection against hybrids (Harrison \& Larson, 2014; Gompert et al., 2017). Species may in particular form clines for traits experiencing divergent selection upon secondary contact, where the slope of a cline reflects the strength of divergent selection (Slatkin, 1973; Barton \& Hewitt, 1985; Gompert et al., 2017). Finally, secondary contact can lead to the coexistence of two isolated species if strong pre- and/or postzygotic barriers exist or evolve (Jiggins, 2000; Canestrelli et al., 2016). The abundance of postglacial lineages or formerly allopatric species in secondary contact has attracted much interest, yet the isolating barriers separating such species often remain unclear (Ortiz-Barrientos et al., 2009; Harrison \& Larson, 2014; Gompert et al., 2017).

Theory suggests that chromosomal rearrangements through fusion and fission of existing chromosomes could act as isolating mechanisms because hybrids are predicted to be at least partially sterile (White, 1978; King, 1995; Dion-Côté \& Barbash, 2017). Fusion and fission events are often widespread in taxonomic groups with holocentric chromosomes, i.e. that lack a centromere, such as Lepidoptera (Melters et al., 2012, de Vos et al., 2020). However, the impact of karyotype evolution on rates of speciation has been challenged given the often widely observed hybridisation and introgression between closely related species despite varying chromosome numbers (Cohuet et al., 2005; Descimon \& Mallet, 2009; Polyakov et al., 2011). Nevertheless, the potential for interspecific gene flow is expected to be less constrained between closely-related species sharing the same number of chromosomes than between species with different chromosome numbers. To prevent costly hybridization, further barriers may subsequently evolve. For example, closely related 
species of Agrodiaetus butterflies evolved increased phenotypic differentiation through reinforcement, allowing for pre-zygotic isolation through mate choice and promoting their coexistence (Lukhtanov et al., 2005). However, other factors may similarly promote reproductive isolation between closely related species. These factors are often speciesdependent and may include, among others, mate choice (Mérot et al., 2017), differences in (micro-) habitat use (Kleckova et al., 2014) or the infection by endosymbionts. The latter has been shown for the cytoplasmically inherited endosymbiotic bacteria Wolbachia, causing uni- or bidirectional cytoplasmatic incompatibility between insect species (Bordenstein et al., 2001; Brucker \& Bordenstein, 2012) or lineages (Zabal-Aguirre et al., 2009). Wolbachia is common, occurring in $\sim 20 \%$ of all insect species (Reuter et al., 2004), yet the outcome of interspecific crosses that bring different Wolbachia lineages together, depends on the host species and may range from no effect to hybrid male sterility or the complete failure to produce viable offspring (Rokas, 2000; Brucker \& Bordenstein, 2012).

With more than one hundred described species, Erebia is one of the most speciesrich Palearctic butterfly genera. Erebia are cold-adapted and mountainous environments represent biodiversity hot-spots (Sonderegger, 2005; Peña et al., 2015). In Europe, Erebia diversified over the last $\sim 15$ million years, with species often differing in their chromosome numbers (Robinson, 1971; Lucek, 2018). This is particularly true for the $E$. tyndarus group, which diversified much more recently, i.e. over the last 0.15-1 million years (Martin et al., 2002; Albre et al., 2008a; Peña et al., 2015) into a total of 10 nominal species and 45 recognised subspecies, often representing different postglacial lineages whose taxonomic status has not been formally assessed (Albre et al., 2008b). The diversification of Erebia and the $E$. tyndarus group in particular has been attributed to changes in chromosome numbers (Lorkovic, 1958; Peña et al., 2015), where rates of speciation were found to be positively correlated with karyological diversity (de Vos et al. 2020). Importantly, changes in chromosome numbers were more frequently anagenetic, i.e. occurring along a branch of the phylogenetic tree than at a speciation event. Novel chromosomes may therefore help to buildup reproductive isolation over time, e.g. by suppressing recombination while some gene flow might still be possible (de Vos et al. 2020).

Species within the E. tyndarus group often form zones of secondary contact with reported cases of hybridisation and interspecific gene flow (Albre et al., 2008a; Descimon \& Mallet, 2009). Three sister species of the E. tyndarus group provide the opportunity to study 
the role of different barriers during secondary postglacial contact: E. tyndarus, E. cassioides and E. nivalis. While E. cassioides has many allopatric populations associated with different mountain ranges from the Cantabrian Mountains in Spain to the Balkans in Eastern Europe (Albre et al., 2008a), the distributions of E. tyndarus and E. nivalis are restricted to the Alps (Figure 1). E. tyndarus occurs in the central Alps excluding E. cassioides, while E. nivalis has its main distribution in the Eastern Alps occurring at higher elevations than E. cassioides. The exception is the narrow contact zone in the Grindelwald region in Switzerland, between an isolated population of $E$. nivalis that persists on the topmost parts of a mountain range, and E. cassioides that occurs in the west, and E. tyndarus in the east (Figure 1; Sonderegger, 2005).

Whereas E. cassioides and E. tyndarus have the same number of chromosomes (haploid $n=10)$, E. nivalis has one chromosome more $(n=11)$. Classic crossing experiments showed that $E$. tyndarus and E. cassioides hybridise readily in the lab but hybrids are only rarely observed in the wild (Lorkovic, 1958; Descimon \& Mallet, 2009), despite the absence of apparent hybrid inviability or sterility (Presgraves, 2002). This is consistent with extrinsic barriers maintaining the species boundaries, but such barriers have not been assessed so far. Crosses between E. tyndarus and E. nivalis have not yet been performed, but intermediates can be found rarely in the wild, while crosses between E. cassioides and E. nivalis produced only a few and sterile F1 offspring (Lorkovic, 1958). Based on restriction site associated sequence (RADseq) data, a former study concluded that interspecific gene flow is absent between the three Erebia species (Gratton et al., 2016). However, this conclusion was based on only a few $(\mathrm{N}=46)$ allopatric individuals, sampled from the entire ranges of the three species, excluding secondary contact zones. The study of secondary contact zones is required to understand interspecific gene flow and the evolutionary mechanisms restricting it (Seehausen et al., 2014).

To test for interspecific gene flow and potential barriers during secondary contact, we studied the only known secondary contact zone between all three species in the Grindelwald region of Switzerland (Figure 1). Given classic crossing experiments (Lorkovic, 1958; Descimon \& Mallet, 2009), we expected interspecific gene flow to be more common between the two sibling species $E$. tyndarus and E. cassioides that have the same karyotype. Conversely, if changes in chromosome numbers act as a barrier to gene flow, we predicted less gene flow between E. nivalis and its sibling species. In the absence of differing 
chromosome numbers and under the assumption that hybridisation is at least to some degree maladaptive and costly, we predicted that other barriers prevent interspecific gene flow between $E$. tyndarus and E. cassioides. The latter may, for example, manifest as phenotypic differentiation, potentially aiding mate recognition or through differences in the utilised habitat to reduce interspecific encounters. Both phenotypic and ecological differentiation may reinforce interspecific differentiation (Gompert et al., 2017; Garner et al., 2018; Butlin \& Smadja, 2018). Lastly, the presence of Wolbachia has been confirmed for Erebia (Gratton et al., 2016). If different Erebia species are infected by different Wolbachia strains, the latter could select against interspecific gene flow by reducing hybrid fitness or even causing hybrid sterility (Rokas, 2000; Brucker \& Bordenstein, 2012). Consequently, we also assessed the prevalence and diversity of Wolbachia, predicting different Wolbachia lineages to be associated with different host species.

\section{Material and Methods}

We collected 95 imagoes belonging to the E. tyndarus species complex during summer 2017 across the previously described contact zone in the Grindelwald area in the Swiss Alps (Sonderegger, 2005). Individuals were a priori assigned by eye to a species based on the location where they were collected and based on phenotypic traits that are commonly used to differentiate between taxa, being mainly the shape and extent of the orange spot on the forewings (Sonderegger, 2005; Figure 1). While clearly belonging to the three focal species, several individuals showed intermediate phenotypes in the contact zone. The sampling of $E$. nivalis was restricted, being a top-priority species on the national red list. All individuals were caught by hand-netting and immediately euthanised with an overdose of ethyl acetate. For each specimen, we recorded its place-of-catch (GPS). The wings of each specimen were clipped and stored in paper bags for further morphological analyses and the body stored in a $-20^{\circ} \mathrm{C}$ freezer prior to DNA extraction. DNA for each specimen was extracted from thorax tissue with the Qiagen Blood \& Tissue Kit (Qiagen, Zug, Switzerland) following the manufacturer's protocol. Individuals were genotyped employing a restriction site associated DNA (RAD) single-end sequencing at Sbfl restriction cut-sites (Baird et al., 2008) on one Illumina HiSeq2500 lane. To increase complexity at the first 10 sequenced base pairs, 10\% bacteriophage PhiX genomic DNA (Illumina Inc., San Diego, CA, USA) was 
added to the run. Library preparation and sequencing was outsourced to Floragenex (Portland, OR, USA).

\section{Data Filtering \& Assembly}

We filtered all sequencing reads to have an intact $S b f 1$ restriction site, followed by de-multiplexing and barcode-trimming with PROCESS_RADTAGS 1.48 (Catchen et al., 2013). Reads without the complete $S b f l$ recognition sequence were subsequently discarded. Using the FASTX toolkit (http://hannonlab.cshl.edu/fastx_toolkit/), we removed reads containing bases with a Phred quality score $<10$ or more than $5 \%$ of base pairs with quality $<30$. This approach yielded $319^{\prime} 072^{\prime} 377$ million high quality reads for analysis.

Given the lack of an Erebia reference genome, we generated a de novo assembly of RAD-tags using all filtered reads for all individuals with USTACKS 1.48 (Catchen et al., 2011) with the following settings: minimum stack size of 100 reads, a maximum of two base pairs of difference for stacks to be merged, excluding loci with unusually high coverage to avoid repetitive regions. The initial de novo assembly consisted of 42'517 contigs. To further identify and remove exogenous contigs from the assembly, we compared the assembly against the NCBI GenBank nucleotide collection with the BLASTN function from BLAST+ 2.7.1 (Camacho et al., 2009). A total of 3188 or $7.0 \%$ of all contigs were of exogenous origin and removed from the initial assembly. We extracted contigs related to Wolbachia (157 contigs) as a separate de novo assembly to further map the prevalence of this parasite across all Erebia specimens (see below). We then mapped the reads for each individual against the cleaned assembly with BWA MEM 0.7.17 (Li, 2013) and used SAMTools 1.7.20 (Li et al., 2009) to filter reads with a mapping quality of $<20$. On average, 2.03 million reads mapped per individual (SD: $\pm 921 \mathrm{k}$ reads; Table S1). We also aligned the raw sequencing reads against the PhiX 174 reference genome (accession: NC_001422; (Sanger et al., 1977)), masking known variants. We then used the PhiX-alignments to create a base-quality score recalibration table with BASERECALIBRATOR from GATK v. 3.7-0 (McKenna et al., 2010). We subsequently recalibrated the base quality scores of each individual to remove potential library effects with the GATK tool PRINTREADS. Lastly, we incorporated a recently published dataset comprising Sbfl based RADseq data for 46 individuals from across the entire range of the $E$. tyndarus complex in the Alps, as well as samples from the Pyrenees and the Apennines for $E$. cassioides (NCBI Sequence Read Archive - SRA, accession SRP065834 from (Gratton et al., 
2016); see Table S1). The dataset by Gratton et al. (2016) also included five individuals from the Grindelwald region (two E. tyndarus and three E. nivalis) as well as three individuals from E. calcaria, which we used as outgroup. We similarly aligned these individuals against the cleaned assembly, applying the same quality filtering settings.

We employed the GatK tool UNIFIEDGenOTYPER to call variants and genotypes in a combined fashion for all individuals, using the following parameters: minimal Phred-scaled base quality score threshold of 20, a genotype likelihood model calling both SNPs and insertions/deletions (indels). Using VCFTOOLs v0.1.14 (Danecek et al., 2011), genotypes with quality $<28$ or depth $<6$ were set to missing. Three individuals with more than $80 \%$ missing data were subsequently removed from the data set. Variants with $>10 \%$ missing genotypes per sampling site, monomorphic sites, SNPs with > 2 alleles, indels and SNPs 10 bp around indels were further removed from the dataset also applying a minor allele frequency cut-off of 0.03 . This filtering step was performed using either all specimens or only for the specimens from the Grindelwald region resulting in 4362 and 4289 polymorphic SNPs, respectively.

\section{Population structure \& gene flow}

To infer the phylogenetic structure across all available specimens, we used RAXML 8.2.11 (Stamatakis, 2014) implementing a generalised time-reversible (GTR) model with optimised substitution rates and a gamma model of rate heterogeneity. We further applied an ascertainment bias correction to account for the fact that we only used polymorphic SNP positions with the AsC_GTRGAMmA function implemented in RAXML. Significance was assessed using 1000 bootstrap replicates followed by a thorough maximum likelihood search. Because hybrid individuals may induce homoplasies in a phylogeny (Seehausen, 2004), we repeated the analysis omitting two identified F1 hybrid individuals (see results) from the analysis.

We inferred population structure in the zone of secondary contact with ADMIXTURE 1.3.0, which uses a likelihood approach to estimate ancestry (Alexander et al., 2009). We ran AdMixtURE multiple times, varying the values for $\mathrm{K}$ assumed populations from 2 to 10 . We then performed a cross-validation test to determine the optimal value of $\mathrm{K}$. We further calculated the pairwise genetic differentiation $\left(G_{S T}\right)$ among the resulting genetic clusters in 
GeNoDIVE v. 2.0b27 (Meirmans \& Van Tienderen, 2004). Significances of $G_{\mathrm{ST}}$ s were assessed with 1000 permutations.

Finally, we tested for patterns of introgression between different Erebia species within the studied contact zone by calculating Patterson's four-taxon D-statistics (Patterson et al., 2012), which compare the frequencies of discordant SNP genealogies. The estimates are based on the so-called ABBA-BABA test, which compares counts of discordant site patterns along a phylogenetic tree. The latter consists of three ingroups and an outgroup, i.e. $(((\mathrm{P} 1, \mathrm{P} 2), \mathrm{P} 3), \mathrm{O})$ with $\mathrm{P} 1$ and $\mathrm{P} 2$ being more closely related and $\mathrm{P} 3$ being more distantlyrelated. In the absence of interspecific gene flow, the number of ABBA and BABA trees should be unbiased and the expected value of Patterson's D-statistic close to zero. The values of D-statistic that are above zero correspond to a higher number of ABBA patterns and thus introgression between P2 and P3, whereas negative values indicate a higher frequency of BABA patterns and introgression between P1 and P3. We performed ABBA/BABA tests using $E$. tyndarus and $E$. nivalis as most closely related sister taxa (i.e. P1 and $\mathrm{P} 2$ ), more distantly related to E. cassioides (P3) and with E. calcaria as outgroup. This scenario reflects the mitochondrial phylogeny (Lucek, 2018) as well as the nuclear relationship when hybrids were excluded (Figure 2). We calculated average $D$ across the genome, its variation across the genome using a jackknife approach and a $Z$ score test to assess if $D$ deviates significantly from zero, following (Martin et al., 2015).

\section{Morphological analyses}

The forewings of Erebia butterflies often differ phenotypically between species in terms of wing shape, the size and extent of the orange spot and the number and position of eyespots (Sonderegger, 2005; Gratton et al., 2016; Figure 1). To capture this phenotypic variation we selected a total of nineteen geometric morphometric landmarks that cover wing shape based on vein intersections or terminations as well as the extent and position of the white, black and orange spots of the right dorsal forewing (Figure S1). Individuals with damaged wings and/or damaged scale patterns were omitted from the analysis. Wings for a total of 72 specimens were digitised using a flatbed scanner. Landmarks were set using TPSDIG2 (Rohlf, 2006), with individuals in random order. We performed Procrustes fits on the obtained landmarks in MORPHOJ 1.06b (Klingenberg, 2011) and extracted the major axis 
of phenotypic variation using a principal component (PC) analysis. We then tested for phenotypic differentiation along PC1 between the genetic groups assigned by ADEGENET using ANOVAs and TukeyHSD post hoc tests.

\section{Testing for niche differentiation}

Several distantly related and coexisting Erebia species have been shown to differ in their microhabitat use (Kleckova et al., 2014), however, to which degree this may also apply to our closely related focal species is unknown. To test for niche differentiation, we extracted the values of a set of environmental variables with a spatial resolution of $25 \mathrm{~m}$ (Guisan et al., 2006) from the GPS recorded locations where each specimen was captured in the field. The variables included were: monthly mean cloudiness (\%), monthly moisture index (1/10mm / month), monthly mean precipitation between 1961-1990 (mm), the monthly mean of average temperature between 1961-1990 (all temperatures in ${ }^{\circ} \mathrm{C}$ ), annual growing degree days for plants (i.e. the number of days with temperature above a threshold of $3^{\circ} \mathrm{C}$ which is critical to growth), maximal temperature in summer and winter, minimal temperature in spring and autumn, annual average site water balance ( $\mathrm{mm} /$ year), number of precipitation days per growing season, the annual average number of frost days during the growing season and lastly, the slope of the terrain $\left({ }^{\circ}\right)$. For monthly-based variables, we used only data for July-September, being the months where the studied Erebia species fly (Sonderegger, 2005). To reduce correlation among variables, we reduced the dataset to three independent principal component (PC) axes accounting for $92.5 \%$ of the total variation (80.4\%, $8.0 \%$ and $4.2 \%$ for PC1-3 respectively, Table S2). We then used NICHEROVER (Swanson et al., 2015) to assess potential multivariate niche differentiation among species based on the retained PC scores. Given the sample sizes, we only compared E. cassioides and E. tyndarus. Each variable was further tested for a difference between the two species using a MANOVA for all traits together and single ANOVAs separately for each trait. All statistical analyses were performed in R 3.5.1 (R Core Team, 2018).

\section{Prevalence of Wolbachia}

Sequences for all individuals from this study and the ones from Gratton et al. (2016) were aligned with BWA MEM against the 157 contigs belonging to Wolbachia, a parasitic 
microbial endosymbiont common to arthropods (Hilgenboecker et al., 2008). Using VCFTOOLS, genotypes with quality $<28$ or depth $<6$ were set to missing and only biallelic SNPs were retained while applying a minor allele frequency cut-off of 0.01 . Only sites with less than $40 \%$ missing data were retained. This approach resulted in a total of 23 polymorphic sites. Unique genotypes were identified, and a haplotype network was constructed using POPART (Leigh \& Bryant, 2015). Individuals with incomplete genotypes were subsequently assigned to the most likely haplotype (see Table S4).

\section{Cline analyses}

We fitted a simple sigmoid cline for a genetic hybrid index between E. cassioides and E. tyndarus based on ADMIXTURE assignment probabilities for $\mathrm{K}=2$ following Westram et al. ( 2018), applying the equations in Derryberry et al. (2013), using maximum likelihood estimation (BBMLE package in R, Bolker 2017). The cline was fitted using either the individual geographic distances or the least cost distances from the westernmost specimen in Figure 3. Further clines were fitted to phenotypic data using PC1 scores, scaled monthly mean precipitation in August and the prevalence of Wolbachia.

\section{Results}

Population genetic structure \& gene flow

Species were well resolved in the RAXML tree, where E. tyndarus and E. nivalis cluster as sister species (Figure 2), consistent with the mitochondrial phylogeny (Peña et al., 2015; Gratton et al., 2016; Lucek, 2018). Due to homoplasy, E. tyndarus and E. cassioides cluster as sister species when the two F1 hybrids between the two species were included (Figure S2).

Three genetic clusters $(\mathrm{K}=3)$, as inferred with ADMIXTURE and representing the three Erebia species, were the best fitting number when using individuals from the contact zone (Figures $3 \&$ S3). Consistent with former reported extents of the contact zone (Sonderegger, 2005 , Figure 1 ) the three species were spatially not randomly distributed, with $E$. tyndarus occurring more in the East and E. cassioides more in the West of the Grindelwald valley, while E. nivalis showed a more limited distribution (Figure 3). ADMIXTURE further suggested interspecific gene flow with two hybrid individuals that were almost equally assigned to $E$. tyndarus and E. cassioides (Figure 3). These two individuals occurred moreover only in a 
narrow zone of secondary contact between the two species. In contrast, three specimens from very distinct parts of the distribution showed evidence consistent with gene flow between $E$. nivalis and the two other species. Levels of genetic differentiation between species as measured with $G_{S T}$ were substantial: $E$. tyndarus vs. $E$. cassioides: $G_{S T}=0.550(95 \%$ confidence interval: 0.538-0.563); E. tyndarus vs. E. nivalis: $G_{S T}=0.454$ (0.439-0.469); $E$. cassioides vs. E. nivalis: $G_{\mathrm{ST}}=0.592(0.579-0.605)$ (all $\left.\mathrm{p}<0.001\right)$.

Average genome-wide $D$ statistics were positive $(D=0.218$; SE: 0.073$)$, indicating an excess of ABBA over BABA sites. This is consistent with $E$. nivalis sharing more genetic variation with $E$. cassioides than with $E$. tyndarus, suggesting interspecific gene flow despite different chromosome numbers. The $Z$ score was $\geq 3$ and thus above the $99 \%$ probability of the underlying standard normal distribution, suggesting a significant deviation of $D$ from zero.

\section{Phenotypic differentiation of wing shapes}

The first PC axis captured $62.3 \%$ of the phenotypic variation (Figure 4 , Table S3), where PC scores differed among species $\left(F_{2,71}=76.4, p<0.001\right)$ with $E$. cassioides individuals being mostly distinct from individuals of the two other species (Tukey post hoc test: both $p<0.001)$. In contrast, $E$. nivalis did not differ from E. tyndarus $(p=0.546)$, but sample size was limited. Individuals differed by the shape and extent of the orange spot, both along the horizontal and vertical wing axis and wing shape, with individuals having more or less condensed wings (Figure 4).

\section{Niche differentiation}

The multivariate niche assessed by NICHEROVER differed between $E$. cassioides and $E$. tyndarus, where E. cassioides showed a $47.1 \%$ overlap with E. tyndarus and E. tyndarus a niche overlap of $56.4 \%$ with E. cassioides. Despite great overlap (Figure 5a), the two species differed statistically along the ecological PC1 axis $\left(F_{1,81}=4.3, p=0.041\right)$, which was driven by differences in temperature and humidity (Table S2). The species similarly differed along PC2 $\left(F_{1,81}=16.6, p<0.001\right)$, which was driven by slope and annual average site water balance, and along PC3 $\left(F_{1,81}=18.2, p<0.001\right)$, explaining variation in humidity. A MANOVA using all environmental variables similarly supported an overall multivariate differentiation between 
the two species $\left(F_{1,81}=11.0, p<0.001\right)$. Testing all variables independently with an ANOVA, while applying a Benjamini-Yekutieli correction for multiple testing, suggested that the environment where the two species occur differs mainly in monthly mean precipitation for months July-September (Figure 5b).

\section{Wolbachia prevalence}

Following filtering, the Wolbachia haplotypes for 59 individuals could be identified (Table S4). Within our studied contact zone, Wolbachia was common in E. cassioides and E. nivalis with a prevalence of $94.4 \%$ and $100 \%$ respectively (Figure 6). In contrast, Wolbachia was absent from E. tyndarus in the contact zone. Three distinct Wolbachia haplotypes occurred (h1-h3), which were differentially distributed among species (Figure 6; Table S4): One Wolbachia haplotype (h1) was exclusively found in an E. calcaria specimen. Haplotype h3 occurred in E. cassioides from the Grindelwald region and the Pyrenees as well as in one E. nivalis specimen from Grindelwald. Conversely, we found a widespread Wolbachia haplotype (h2), occurring in E. cassioides from Italy and Austria, in all but one E. nivalis and in the one E. tyndarus with an identifiable Wolbachia haplotype that was sampled by Gratton et al. (2016) from the Eastern part of Switzerland. Removing the missing data threshold for filtering suggested that Wolbachia occurred in three additional E. tyndarus individuals from the Italian Alps (Table S4), but given the high amount of missing data, Wolbachia could not be unambiguously assigned to a given haplotype. Lastly, the two F1 hybrids between E. cassioides and E. tyndarus (Figures $2 \& 3$ ) had the Wolbachia haplotype found in E. cassioides from Grindelwald (h2).

\section{Cline analyses}

Using geographic distances, the segregation between $E$. cassioides and $E$. tyndarus fell along a steep genetic cline where the cline centre was close to the position of the two F1 hybrids (centre position: $4.64 \mathrm{~km}$ [95\% Cl: 4.63-4.64], Figure 7a). The estimates for the cline centres for both the Wolbachia prevalence and wing morphology overlapped with the genetic cline (Wolbachia: 4.74 km [95\% Cl: 4.35-4.97]; wing morphology: 4.57 km [95\% Cl: 3.90-4.99], Figure $7 \mathrm{~b} \& \mathrm{c})$. Precipitation did show a clinal variation across the transect; however, its centre was shifted to the east $(6.15 \mathrm{~km}$ [95\% Cl: 6.10-6.19], Figure $7 \mathrm{~d})$. The cline analyses based on least-cost distances similarly suggested an overlap between the cline centre for 
the genetic data (6.64 km [95\% Cl: 6.63-6.65]; Figure S4) and the centres for Wolbachia (6.77 km [95\% Cl: 6.27-7.16]) and morphology (6.50 km [95\% Cl: 5.49-7.04]), while a shift occurs for precipitation (9.25 km [95\% Cl: 9.03-9.48]).

\section{Discussion}

Zones of secondary contact have gathered much theoretical and empirical interest to study the process of speciation (Barton \& Hewitt, 1985; Kirkpatrick, 2001; Servedio \& Noor, 2003; Ortiz-Barrientos et al., 2009; Butlin \& Smadja, 2018). However, the multivariate intrinsic and extrinsic factors that shape such contact zones, and that may prevent interspecific gene flow are often unknown (Harrison \& Larson, 2014; Gompert et al., 2017). Here, we studied such a secondary contact zone, combining population genomic with phenotypic and ecological data. We show that gene flow can occur across the species boundaries despite varying chromosome numbers, but that the potential for gene flow likely differs between species comparisons (Figure 3). The latter likely reflects different evolutionary mechanisms being at play. While some interspecific gene flow between the three Erebia species is consistent with former phenotypic and observational data from the wild (Sonderegger, 2005; Descimon \& Mallet, 2009), our findings suggest that the species boundaries are more dynamic than found in a recent genomic study on the same species complex and whose data have been incorporated in this study (Gratton et al., 2016). This is because Gratton et al. (2016) studied only a few, mostly allopatric individuals from across the entire ranges of the studied Erebia species where interspecific gene flow could not be detected.

While hybrids between species with different karyotypes are often predicted to yield offspring that are somewhat sterile (White, 1978; King, 1995; Dion-Côté \& Barbash, 2017), we observed introgression despite such differences, i.e. current gene flow between $E$. nivalis and its two sibling species (Figure 3 ). The excess of ABBA over BABA sites is further consistent with past gene flow, where gene flow was higher from E. cassioides to E. nivalis than to E. tyndarus. Meiosis may still be possible between closely related species despite varying chromosome number (Searle, 1998; Lukhtanov et al., 2018). However, the extent of introgression between $E$. nivalis and its sibling species is limited as indicated by the Admixture analysis (Figure 3), suggesting the presence of strong isolating mechanisms. Gene flow between $E$. nivalis and $E$. cassioides contrasts with evidence from classic crossing 
experiments, where interspecific crosses were only possible in one direction, producing very few and sterile offspring in the lab (Lorkovic, 1958). Conversely, crosses between the closely-related E. tyndarus and E. cassioides that have the same karyotype, were found to readily produce offspring in the same study, consistent with the absence of inviability or sterility (Lorkovic, 1958; Presgraves, 2002). Contrary to these experimental crosses, we found interspecific hybridisation between E. tyndarus and E. cassioides to manifest solely as few F1 hybrids in our studied contact zone, in the absence of further introgression (Figures 3), resulting in a steep genomic cline separating the two species (Figure 7a). This suggests the presence of postzygotic incompatibilities or strong selection against hybrids. Although only few admixed individuals were found, the estimated cline may differ only little from an actual cline given the already relatively dense sampling and the small geographic range of this contact zone.

Hybrid adults may be absent due to pre-zygotic barriers, intrinsic incompatibilities or natural selection against hybrids imposed by the environment. Ecological differences between co-occurring Erebia species that could lead to habitat-dependent divergent selection and thus selection against hybrids, are often subtle in Erebia and linked to different microhabitat use even at a very small geographic scale (Kleckova et al., 2014). The multivariate ecological niches of $E$. tyndarus and E. cassioides overlap to a large extent (Figure 5), differing mainly in mean monthly precipitation. However, this difference was consistent among the months of the main flight period of imagoes in the $E$. tyndarus complex (Sonderegger, 2005). Moreover, the observed difference in precipitation is consistent with the average ecological differentiation between the studied Erebia species across the entire species range (Schweiger et al., 2014). Natural selection against hybrids could also act during the larval stage, which takes two years in the three Erebia species studied here (Lorkovic, 1958; Sonderegger, 2005). However, unlike for imagoes, little is known about the ecological niche of the different larval stages, where the very few existing observational data does not allow to determine if Erebia species differ, e.g., in their larval microhabitat or their host plant preference (Sonderegger, 2005; Albre et al., 2008b). Although precipitation fell along a geographic cline, the latter did not coincide with the genomic cline but was shifted to the east (Figure 7d), suggesting that habitat-dependent selection is less likely to be the key driver of differentiation in this system.

This article is protected by copyright. All rights reserved 
Differences in wing shape and colour patterns are common targets for mate choice in butterflies and thus a target for selection and the evolution of pre-zygotic isolation (Kemp, 2007; Finkbeiner et al., 2014). E. tyndarus and E. cassioides are known to differ in both traits (Sonderegger, 2005; Gratton et al., 2016) and consistently we found E. cassioides to show more elongated wings and reduced orange spots than E. tyndarus (Figure 4). The variation along the leading multivariate phenotypic axis fell along a cline for which the 95\% confidence interval of the centre estimate overlapped with the genetic cline (Figure 7c). While the observed phenotypic differentiation could primarily reflect divergence in allopatry, reinforcement, whereby differentiation would be increased in zones of secondary contact compared to allopatric populations, may also be at play (Gompert et al., 2017; Butlin \& Smadja, 2018). Sonderegger (2005) indeed showed that E. cassioides becomes more tyndarus-like with increasing distance from the studied contact zone, i.e., exhibiting an increased orange coloration on the forewing. If, and to what degree reinforcement may be at play requires further investigation incorporating more allopatric populations.

Two different strains of the endosymbiotic bacteria Wolbachia were found among individuals of the $E$. tyndarus complex in the Grindelwald region - occurring predominantly in E. cassioides (haplotype h3) and E. nivalis (h2), while E. tyndarus showed no infection in the contact zone (Figure 6, Table S4). Because different Wolbachia infections can reduce hybrid fitness and even cause sterility in other species through cytoplasmic incompatibility (Rokas, 2000; Brucker \& Bordenstein, 2012), the observed prevalence of Wolbachia may account for the observed lack of interspecific gene flow between $E$. tyndarus and $E$. cassioides in the contact zone. Indeed, both F1 hybrids between the aforementioned species carry the Wolbachia haplotype found in E. cassioides, and as a result, the Wolbachia prevalence falls along a cline that overlaps with the genomic cline (Figure 7b). The two Wolbachia strains are likely old, i.e. diverged in allopatry in different glacial refugia. This is because the haplotype $h 3$ is restricted to $E$. cassioides from the western part of the species distribution, including the Grindelwald region and the Pyrenees. whereas $h 2$ occurs in $E$. cassioides from a south-eastern refugium (Italy) as well as in the sister species $E$. nivalis and E. tyndarus from the same glacial refugium (Schmitt et al., 2016). The crossing experiments conducted by Lorkovic (1958) were performed using E. cassioides from the Eastern Alps and thus between individuals that were exposed to the same Wolbachia strain, which could explain why E. tyndarus and E. cassioides could be crossed. E. tyndarus can be infected by 
Wolbachia but the prevalence is much lower than in the two other species. It seems therefore as if E. tyndarus mostly lost Wolbachia, which can occur during the colonisation of a new environment through drift or altered selective regimes (Reuter et al., 2004), such as during a postglacial range expansion. Further experimental work is thus needed to elucidate the role of Wolbachia as a barrier to gene flow.

\section{Conclusions}

Using population genomic data, we showed that some interspecific gene flow may still be possible between closely-related species despite karyological variation in the recently-evolved E. tyndarus complex. Changes in chromosome numbers may therefore represent a strong, but incomplete and only one of several barriers to gene flow in zones of secondary contact (Figure 3). Surprisingly though, the species form very narrow contact zones where hybridisation seems to be limited, i.e. resulting solely in F1 hybrids between the two species having the same karyotype, which contradicts classic crossing experiments (Lorkovic, 1958). The lack of subsequent later-generation introgression likely reflects the interplay of additional barriers to gene flow. Based on cline analyses, we identified two potential targets of divergent selection: phenotypic differentiation that could lead to prezygotic isolation and differences in the rates of infection of the endosymbiont Wolbachia. These results highlight that there are many evolutionary mechanisms at play in zones of secondary contact, whose strengths and sequence may vary and need to be assessed in detail (Lackey \& Boughman, 2017; Mérot et al., 2017).

\section{Acknowledgement}

\section{Data Accessibility Statement}

Genomic data will be archived on the NCBI Short Read Archive. Phenotypic and ecological data will be archived on DRYAD. 


\section{References}

Albre, J., Gers, C. \& Legal, L. (2008a). Molecular phylogeny of the Erebia tyndarus (Lepidoptera, Rhopalocera, Nymphalidae, Satyrinae) species group combining CoxII and ND5 mitochondrial genes: a case study of a recent radiation. Molecular Phylogenetics and Evolution, 47, 196-210.

Albre, J., Gers, C. \& Legal, L. (2008b). Taxonomic notes on the species of the Erebia tyndarus group (Lepidoptera, Nymphalidae, Satyrinae). Lépidoptères, 17, 12-28.

Alexander, D.H., Novembre, J. \& Lange, K. (2009). Fast model-based estimation of ancestry in unrelated individuals. Genome Research, 19, 1655-1664.

Baird, N.A., Etter, P.D., Atwood, T.S., Currey, M.C., Shiver, A.L., Lewis, Z.A., et al. (2008). Rapid SNP discovery and genetic mapping using sequenced RAD markers. PLOS ONE, 3, e3376.

Barton, N.J. \& Hewitt, G.M. (1985). Analysis of hybrid zones. Annual Review of Ecology, Evolution, and Systematics, 16 113-148.

Bordenstein, S.R., O'Hara, F.P. \& Werren, J.H. (2001). Wolbachia-induced incompatibility precedes other hybrid incompatibilities in Nasonia. Nature, 409, 707-710.

Brucker, R.M. \& Bordenstein, S.R. (2012). Speciation by symbiosis. Trends in Ecology and Evolution, 27, 443-451.

Butlin, R.K. \& Smadja, C.M. (2018). Coupling, reinforcement, and speciation. American Naturalist, 191, 155-172.

Camacho, C., Coulouris, G., Avagyan, V., Ma, N., Papadopoulos, J., Bealer, K., et al. (2009). BLAST+: architecture and applications. BMC Bioinformatics, 10, 421.

Canestrelli, D., Porretta, D., Lowe, W.H., Bisconti, R., Carere, C. \& Nascetti, G. (2016). The tangled evolutionary legacies of range expansion and hybridization. Trends in Ecology and Evolution, 31, 677-688.

Catchen, J., Hohenlohe, P.A., Bassham, S., Amores, A. \& Cresko, W.A. (2013). Stacks: an 
analysis tool set for population genomics. Molecular Ecology, 22, 3124-3140.

Catchen, J.M., Amores, A., Hohenlohe, P., Cresko, W. \& Postlethwait, J.H. (2011). Stacks: building and genotyping Loci de novo from short-read sequences. G3, 1, 171-182.

Cohuet, A., Dia, I., Simard, F., Raymond, M., Rousset, F., Antonio-Nkondjio, C., et al. (2005). Gene flow between chromosomal forms of the malaria vector Anopheles funestus in Cameroon, Central Africa, and its relevance in malaria fighting. Genetics, 169, 301-311.

Danecek, P., Auton, A., Abecasis, G., Albers, C.A., Banks, E., DePristo, M.A., et al. (2011). The variant call format and VCFtools. Bioinformatics, 27, 2156-2158.

de Vos, J.M., Augustijnen, H., Bätscher, L. \& Lucek, K. (2020). Speciation through chromosomal fusion and fission in Lepidoptera. Philosophical Transactions of the Royal Society $\mathrm{B}$, in press.

Derryberry, E.P., Derryberry, G.E., Maley, J.M. \& Brumfield, R.T. (2013). hzar: hybrid zone analysis using an R software package. Molecular Ecology Resources, 14, 652-663.

Descimon, H. \& Mallet, J. (2009). Bad species. In: Ecology of butterflies in Europe, Cambridge University Press, Cambridge, UK.

Dion-Côté, A.-M. \& Barbash, D.A. (2017). Beyond speciation genes: an overview of genome stability in evolution and speciation. Current Opinion in Genetics \& Development, 47, $17-23$.

Finkbeiner, S.D., Briscoe, A.D. \& Reed, R.D. (2014). Warning signals are seductive: Relative contributions of color and pattern to predator avoidance and mate attraction in Heliconius butterflies. Evolution, 68, 3410-3420.

Garner, A., Goulet, B., Farnitano, M., Molina-Henao, Y. \& Hopkins, R. (2018). Genomic signatures of reinforcement. Genes, 9, 191.

Gompert, Z., Mandeville, E.G. \& Buerkle, C.A. (2017). Analysis of population genomic data from hybrid zones. Annual Review of Ecology, Evolution, and Systematics, 48, 207-229.

Gratton, P., Trucchi, E., Trasatti, A., Riccarducci, G., Marta, S., Allegrucci, G., ... Sbordoni, V. 
(2016). Testing classical species properties with contemporary data: how "bad species" in the brassy ringlets (Erebia tyndarus complex, Lepidoptera) turned good. Systematic Biology, 65, 292-303.

Guisan, A., Broennimann, O., Engler, R., Vust, M., Yoccoz, N.G., Lehmann, A., Zimmermann, N. E. (2006). Using niche-based models to improve the sampling of rare species. Conservation Biology, 20, 501-511.

Harrison, R.G. \& Larson, E.L. (2014). Hybridisation, introgression, and the nature of species boundaries. Journal of Heredity, 105 Suppl 1, 795-809.

Hewitt, G. (2000). The genetic legacy of the Quaternary ice ages. Nature, 405, 907-913.

Hilgenboecker, K., Hammerstein, P., Schlattmann, P., Telschow, A. \& Werren, J.H. (2008). How many species are infected with Wolbachia? A statistical analysis of current data. FEMS Microbiology Letters, 281, 215-220.

Jiggins, C.D \& Mallet, J. (2000). Bimodal hybrid zones and speciation. Trends in Ecology and Evolution, 15, 250-255.

Kemp, D.J. (2007). Female butterflies prefer males bearing bright iridescent ornamentation. Proceedings of the Royal Society B, 274, 1043-1047.

King, M. (1995). Species Evolution. Cambridge University Press, Cambridge, UK.

Kirkpatrick, M. (2001). Reinforcement during ecological speciation. Proceedings of the Royal Society B, 268, 1259-1263.

Kleckova, I., Konvicka, M. \& Klecka, J. (2014). Thermoregulation and microhabitat use in mountain butterflies of the genus Erebia: importance of fine-scale habitat heterogeneity. Journal of Thermal Biology, 41, 50-58.

Klingenberg, C.P. (2011). MorphoJ: an integrated software package for geometric morphometrics. Molecular Ecology Resources, 11, 353-357.

Lackey, A.C.R. \& Boughman, J.W. (2017). Evolution of reproductive isolation in stickleback fish. Evolution, 71, 357-372.

This article is protected by copyright. All rights reserved 
Leigh, J.W. \& Bryant, D. (2015). popart: full-feature software for haplotype network construction. Methods in Ecology and Evolution, 6, 1110-1116.

Li, H. (2013). Aligning sequence reads, clone sequences and assembly contigs with BWAMEM. arXiv.org.

Li, H., Handsaker, B., Wysoker, A., Fennell, T., Ruan, J., Homer, N., ... 1000 Genome Project Data Processing Subgroup. (2009). The Sequence Alignment/Map format and SAMtools. Bioinformatics, 25, 2078-2079.

Lorkovic, Z. (1958). Some peculiarities of spatially and sexually restricted gene exchange in the Erebia tyndarus group. Cold Spring Harbor Symposium in Quantitative Biology, 23, 319-325.

Lucek, K. (2018). Evolutionary mechanisms of varying chromosome numbers in the radiation of Erebia butterflies. Genes 9, 1-9.

Lukhtanov, V.A., Dinca, V., Friberg, M., Šíchová, J., Olofsson, M., Vila, R. ... Wiklund, C. (2018). Versatility of multivalent orientation, inverted meiosis, and rescued fitness in holocentric chromosomal hybrids. Proceedings of the National Academy of Sciences USA, 115, E9610-E9619.

Lukhtanov, V.A., Kandul, N.P., Plotkin, J.B., Dantchenko, A.V., Haig, D. \& Pierce, N.E. (2005). Reinforcement of pre-zygotic isolation and karyotype evolution in Agrodiaetus butterflies. Nature, 436, 385-389.

Marie Curie SPECIATION Network (2012). What do we need to know about speciation? Trends in Ecology and Evolution, 27, 27-39.

Martin, J.F., Gilles, A., Lortscher, M. \& Descimon, H. (2002). Phylogenetics and differentiation among the western taxa of the Erebia tyndarus group (Lepidoptera : Nymphalidae). Biological Journal of the Linnean Society, 75: 319-332.

Martin, S.H., Davey, J.W. \& Jiggins, C.D. (2015). Evaluating the use of ABBA-BABA statistics to locate introgressed loci. Molecular Biology and Evolution, 32, 244-257. 
McKenna, A., Hanna, M., Banks, E., Sivachenko, A., Cibulskis, K., Kernytsky, A., ... DePristo, MA. (2010). The Genome Analysis Toolkit: a MapReduce framework for analysing nextgeneration DNA sequencing data. Genome Research, 20, 1297-1303.

Meirmans, P.G. \& Van Tienderen, P. (2004). GENOTYPE and GENODIVE: two programs for the analysis of genetic diversity of asexual organisms. Molecular Ecology Notes, 4, 792794.

Melters, D.P., Paliulis, L.V., Korf, I.F. \& Chan, S.W.L. (2012). Holocentric chromosomes: convergent evolution, meiotic adaptations, and genomic analysis. Chromosome Research, 20, 579-593.

Mérot, C., Salazar, C., Merrill, R.M., Jiggins, C.D. \& Joron, M. (2017). What shapes the continuum of reproductive isolation? Lessons from Heliconius butterflies. Proceedings of the Royal Society B, 284, 20170335.

Ortiz-Barrientos, D., Grealy, A. \& Nosil, P. (2009). The genetics and ecology of reinforcement: implications for the evolution of prezygotic isolation in sympatry and beyond. Annals of the New York Academy of Sciences, 1168, 156-182.

Patterson, N., Moorjani, P., Luo, Y., Mallick, S., Rohland, N., Zhan, Y. ... Reich, D. (2012). Ancient admixture in human history. Genetics, 192, 1065-1093.

Peña, C., Witthauer, H., Kleckova, I. \& Fric, Z. (2015). Adaptive radiations in butterflies: evolutionary history of the genus Erebia (Nymphalidae: Satyrinae). Biological Journal of the Linnean Society, 116, 449-467.

Polyakov, A.V., White, T.A., Jones, R.M., Borodin, P.M. \& Searle, J.B. (2011). Natural hybridization between extremely divergent chromosomal races of the common shrew (Sorex araneus, Soricidae, Soricomorpha): hybrid zone in Siberia. Journal of Evolutionary Biology, 24, 1393-1402.

Presgraves, D.C. (2002). Patterns of postzygotic isolation in Lepidoptera. Evolution, 56, 1168-1183.

Reuter, M., Pedersen, J.S. \& Keller, L. (2004). Loss of Wolbachia infection during colonisation 
in the invasive Argentine ant Linepithema humile. Proceedings of the Royal Society B, $267,1277-1285$.

Robinson, R. (1971). Lepidoptera Genetics, Pergamon Press Inc, Oxford, UK.

Rohlf, F. J. (2006). Version 2.10. Stony Brook, NY: Department of Ecology and Evolution, State University.

Rokas, I. (2000). Wolbachia as a speciation agent. Trends in Ecology and Evolution, 15, 4445.

Sanger, F., Air, G.M., Barrell, B.G., Brown, N.L., Coulson, A.R., Fiddes, C.A. ... Smith, M. (1977). Nucleotide sequence of bacteriophage phi X174 DNA. Nature, 265, 687-695.

Schmitt, T., Louy, D., Zimmermann, E. \& Habel, J.C. (2016). Species radiation in the Alps: multiple range shifts caused diversification in ringlet butterflies in the European high mountains. Organisms Diversity \& Evolution, 16, 791-808.

Schweiger, O., Harpke, A., Wiemers, M. \& Settele, J. (2014). CLIMBER: Climatic niche characteristics of the butterflies in Europe. Zookeys, 65-84.

Searle, J.B. (1998). Speciation, chromosomes, and genomes. Genome Research, 8: 1-3.

Seehausen, O. (2004). Hybridization and adaptive radiation. Trends in Ecology and Evolution, 19, 198-207.

Seehausen, O., Butlin, R.K., Keller, I., Wagner, C.E., Boughman, J.W., Hohenlohe, P.A. ... Widmer A. (2014). Genomics and the origin of species. Nature Review Genetics, 15: 176-192.

Servedio, M.R. \& Noor, M.A.F. (2003). The role of reinforcement in speciation: theory and data. Annual Review of Ecology, Evolution, and Systematics, 34, 339-364.

Slatkin, M. (1973). Gene flow and selection in a cline. Genetics, 75, 733-756.

Sonderegger, P. (2005). Die Erebien der Schweiz. Peter Sonderegger, Brügg bei Biel, Switzerland. 
Stamatakis, A. (2014). RAxML version 8: a tool for phylogenetic analysis and post-analysis of large phylogenies. Bioinformatics, 30, 1312-1313.

Swanson, H.K., Lysy, M., Power, M., Stasko, A.D., Johnson, J.D. \& Reist, J.D. (2015). A new probabilistic method for quantifying n-dimensional ecological niches and niche overlap. Ecology, 96, 318-324.

Westram, A.M., Rafajlović, M., Chaube, P., Faria, R., Larsson, T., Panova, M. ... Butlin, R. (2018). Clines on the seashore: The genomic architecture underlying rapid divergence in the face of gene flow. Evolution Letters, 2, 297-309.

White, M.J.D. (1978). Modes of Speciation, W. H. Freeman and Co., San Francisco, CA, USA.

Zabal-Aguirre, M., Arroyo, F. \& Bella, J.L. (2009). Distribution of Wolbachia infection in Chorthippus parallelus populations within and beyonda Pyrenean hybrid zone. Heredity, 104, 174-184. 


\section{Figure Legends}

Figure 1: Elevation map of the Alps in central Europe (modified from Wikimedia). Following Sonderegger (2005), the ranges of the three focal species E. cassioides (green), E. tyndarus (blue) and E. nivalis (orange) are indicated. The centre of the black square further indicates the location of the studied contact zone in the Grindelwald area. For each species, the picture of a mounted specimen collected in the contact zone by Peter Sonderegger is shown.

Figure 2: Phylogram artificially rooted on the node that separates $E$. calcaria from the other species of the $E$. tyndarus complex. Red dots represent nodes with $>95 \%$ bootstrap support. Circles represent samples from the Grindelwald area, triangles samples from elsewhere, i.e. AT - Austria, CH - Switzerland, FR - France, IT - Italy (from Gratton et al. 2016). Colours indicate species.

Figure 3: Individual-based assignments using Admixture for samples from the Grindelwald region. The case for three genetic clusters $(K=3)$ is shown as a bar diagram (top) and as pies plotted on the map (bottom; (C) OpenStreetMap Project 2018).

Figure 4: Bar plot summarising phenotypic variation for wing shape and the shape of the orange spot along the leading principal component (PC) axis explaining $62.3 \%$ of all phenotypic variation.

Figure 5: Niche variation between E. cassioides (green) and E. tyndarus (blue). a) Principal component (PC) scores based on all ecological data. b) Boxplots summarising monthly mean precipitation (in $\mathrm{mm}$ ) for July-September, i.e. the months where imagoes occur. Significance levels are based on ANOVAs.

Figure 6: Wolbachia strains and prevalence among Erebia. a) median joining network for the three Wolbachia haplotypes $(h 1, h 2, h 3)$ found in 59 Erebia specimens. Indicated are the countries where haplotypes were found, i.e. AT - Austria, CH - Switzerland, FR - France, IT Italy, SLO - Slovenia. Dashed lines indicate the number of substitution differences. b) 
Wolbachia prevalence across the sampled range in Grindelwald. Two Wolbachia genotypes are indicated ( $h 2:$ square, $h 3$ : circle). Colours indicate species assignments from Admixture (see Figure 3). For $h 3$, the black area in a) represent the two F1 hybrids. Wolbachia was absent in the E. tyndarus samples (see Table S4).

Figure 7: Clines between E. cassioides (green) and E. tyndarus (blue) along a west-east transect for a) genomic data, b) Wolbachia prevalence, c) PC1 scores of wing morphology, d) scaled precipitation in August. The black dots indicate the two F1 hybrids. The orange line represents the fitted cline, the black bar the cline centre with the respective $95 \%$ confidence interval in grey.

Figure S1: Upper forewing showing the landmarks used for the geometric morphometric analysis: LM1 - base of wing, LM2 - intersection of the cell and the second term, LM3 intersection of the cell and the third term, LM4 - intersection of the cell and the fourth term, LM5-9: outer margins of the second to sixth termen, LM10 outer margin of the apex, LM11 upper extent of upper black spot, LM12 inner upper extent of upper black spot, LM13 centre of the white spot in the upper black spot, LM14 centre of the white spot in the lower black spot, LM15 inner lower extent of lower black spot, LM16 lower extent of lower black spot, LM17 lower extent of orange cell, LM18 basal extent of orange cell, LM19 upper extent of orange cell. LM11-16 were set along a line that is parallel to the extent of the orange cell.

Figure S2: Phylogram artificially rooted on the node that separates E. calcaria from the other species of the E. tyndarus complex. The two $\mathrm{F} 1$ hybrids between $E$. tyndarus and $E$. cassioides were retained in this analysis (see Figure 1). Grey dots represent nodes with $>95 \%$ bootstrap support. Circles represent samples from the Grindelwald area, triangles samples from elsewhere, i.e. AT - Austria, $\mathrm{CH}$ - Switzerland, FR - France, IT - Italy (from Gratton et al. 2016). Colours indicate species. 
Figure S3: Cross validation error from Admixture when assuming 1-10 genetic clusters (K).

Figure S4: Clines fitted on least cost distances along a west-east transect for a) genomic data, b) Wolbachia prevalence, c) PC1 scores of wing morphology, d) scaled precipitation in August. The black dots indicate the two F1 hybrids. The orange line represents the fitted cline, the black bar the cline centre with the respective $95 \%$ confidence interval in grey. 


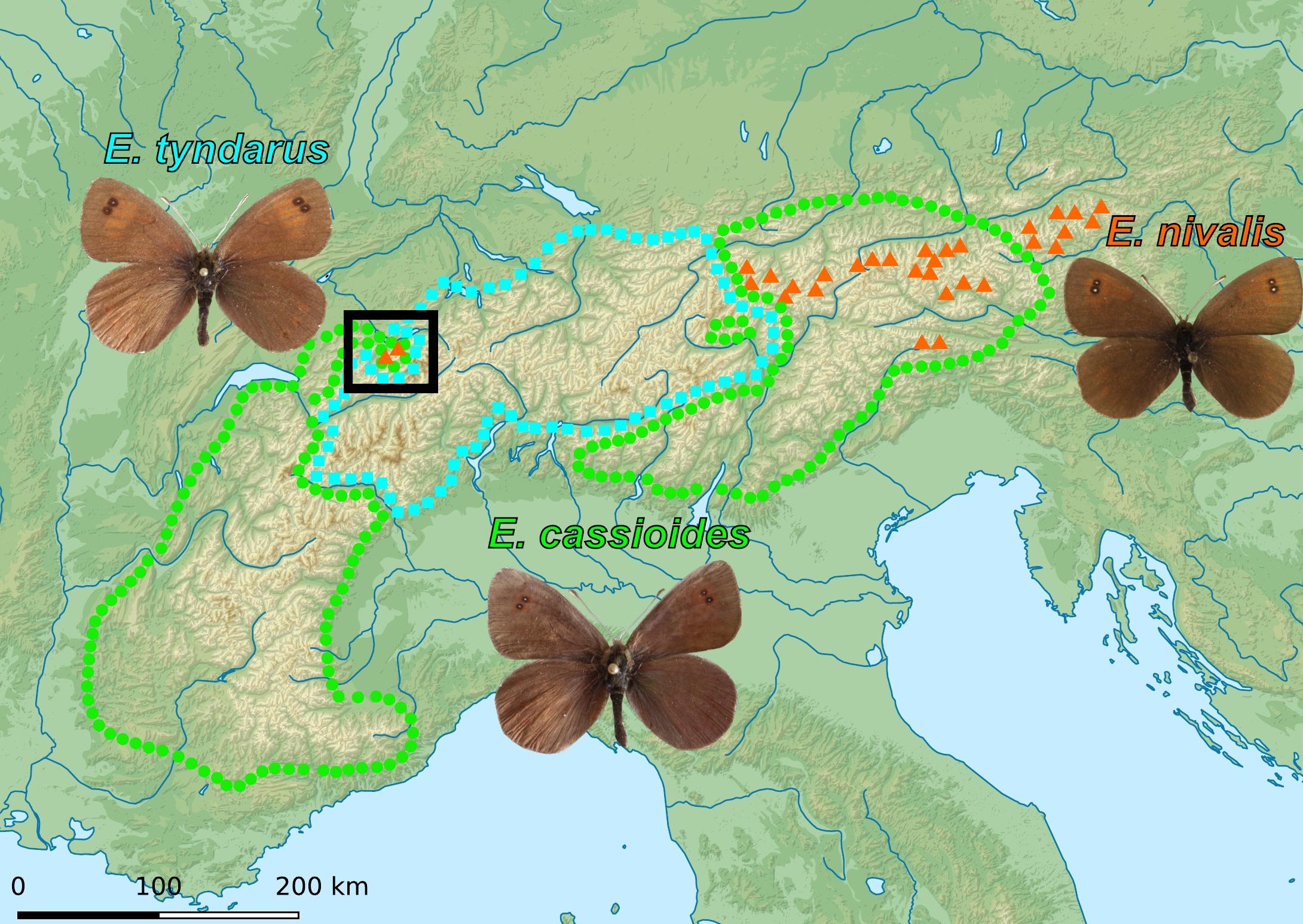




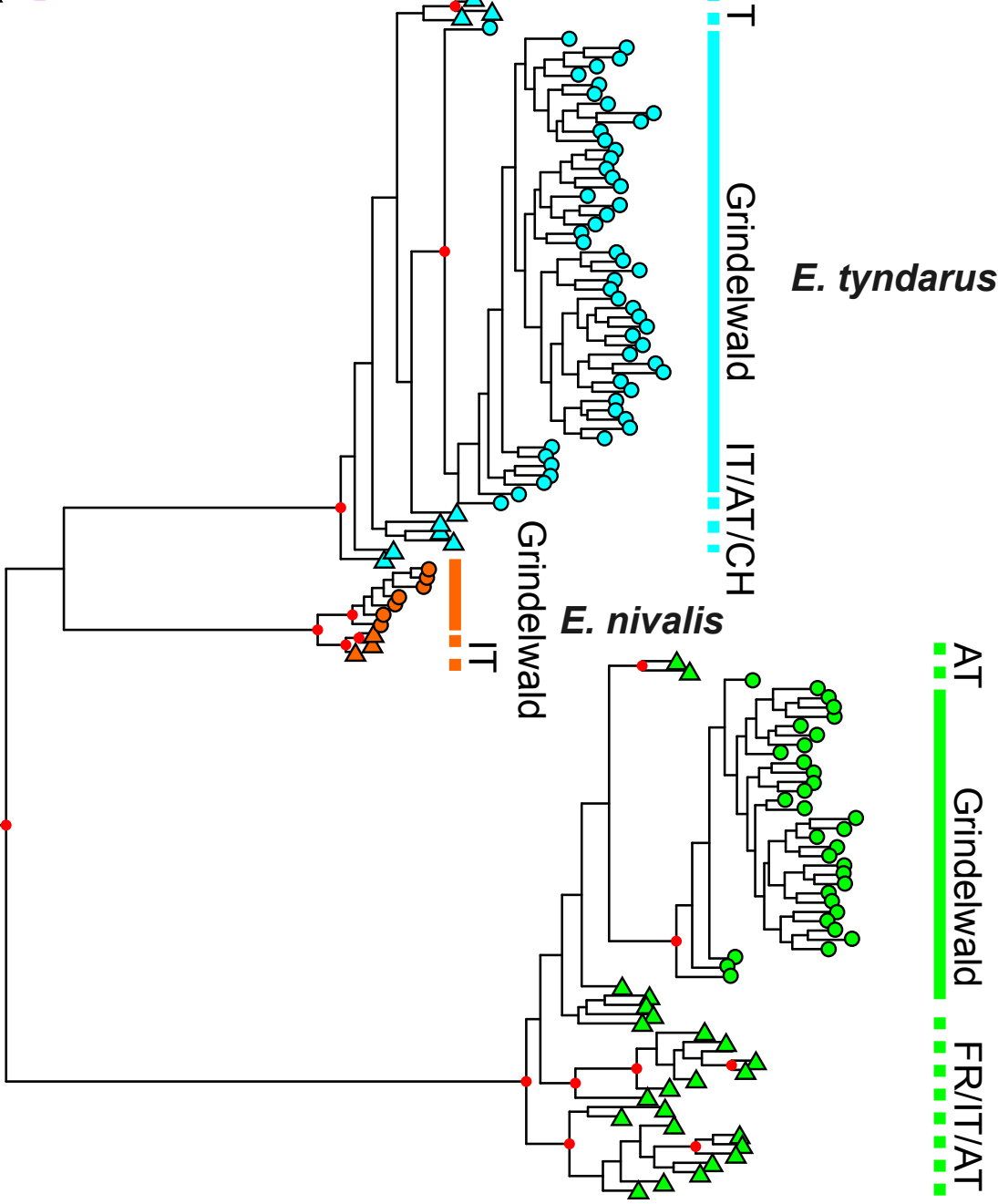




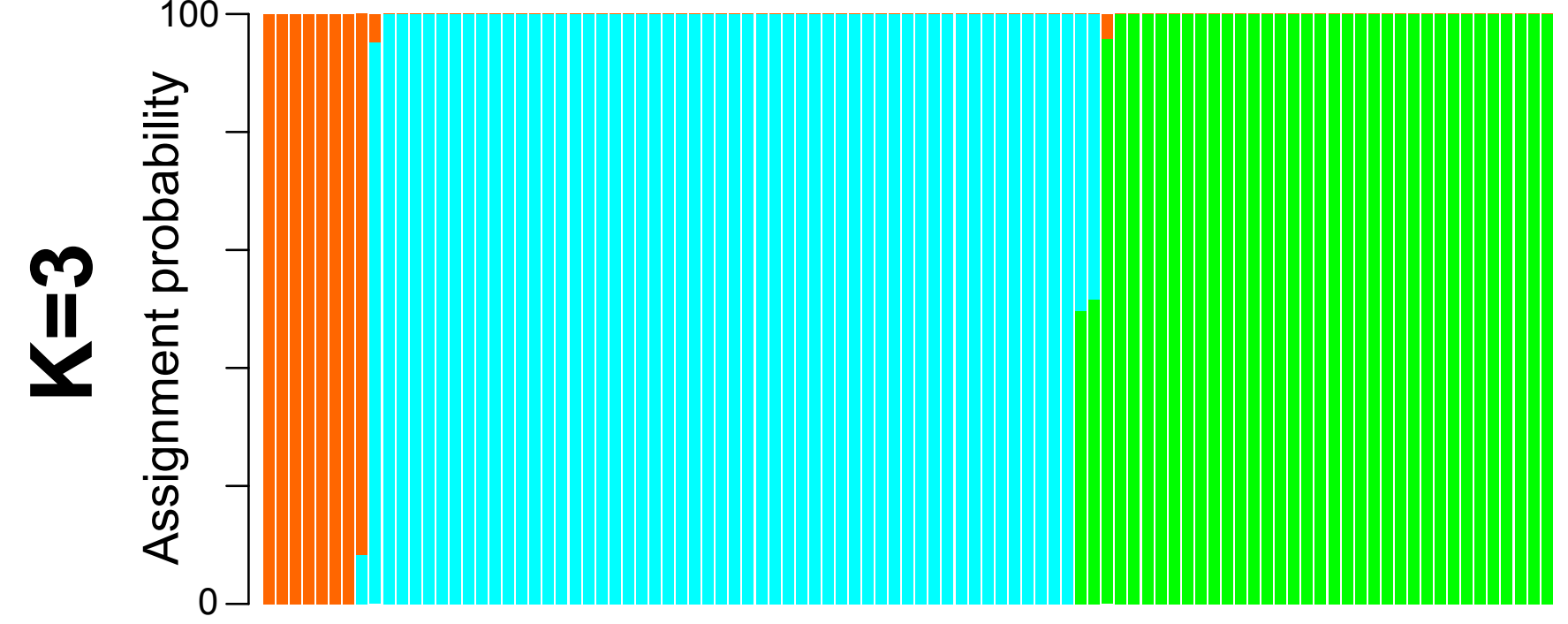

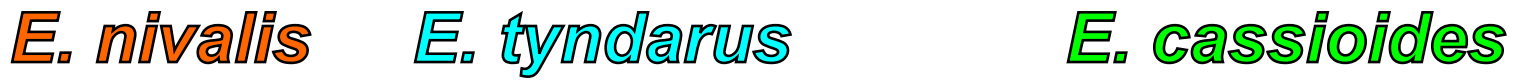

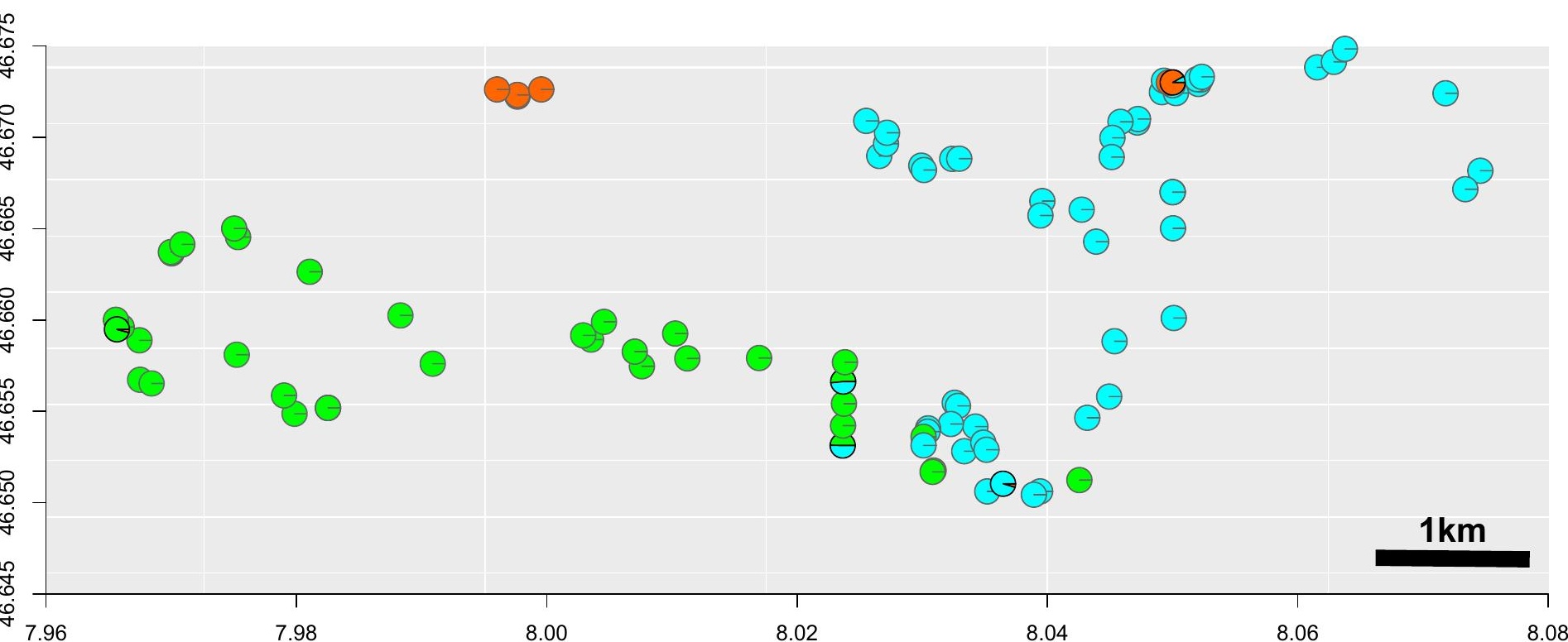



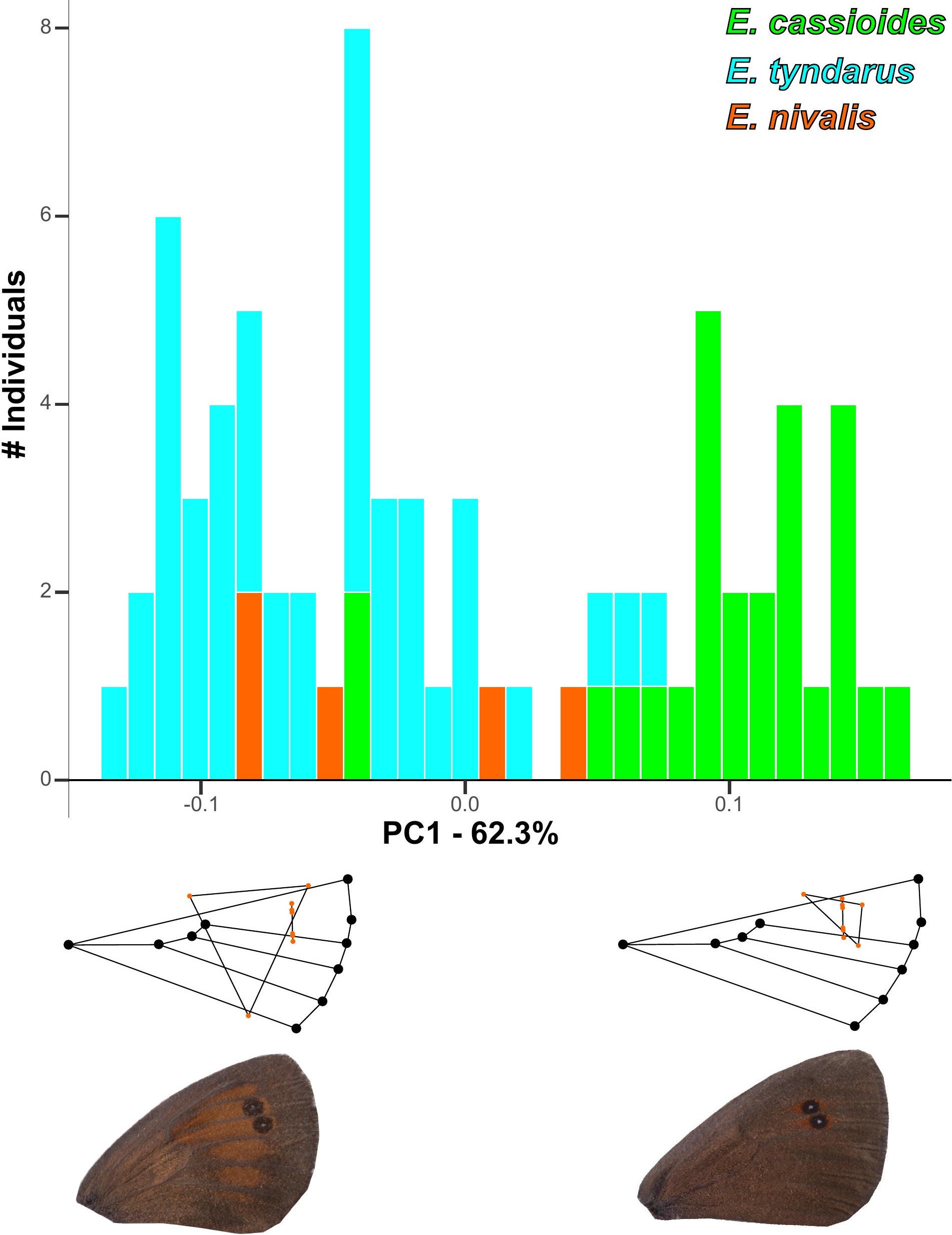


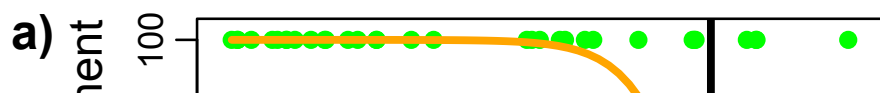

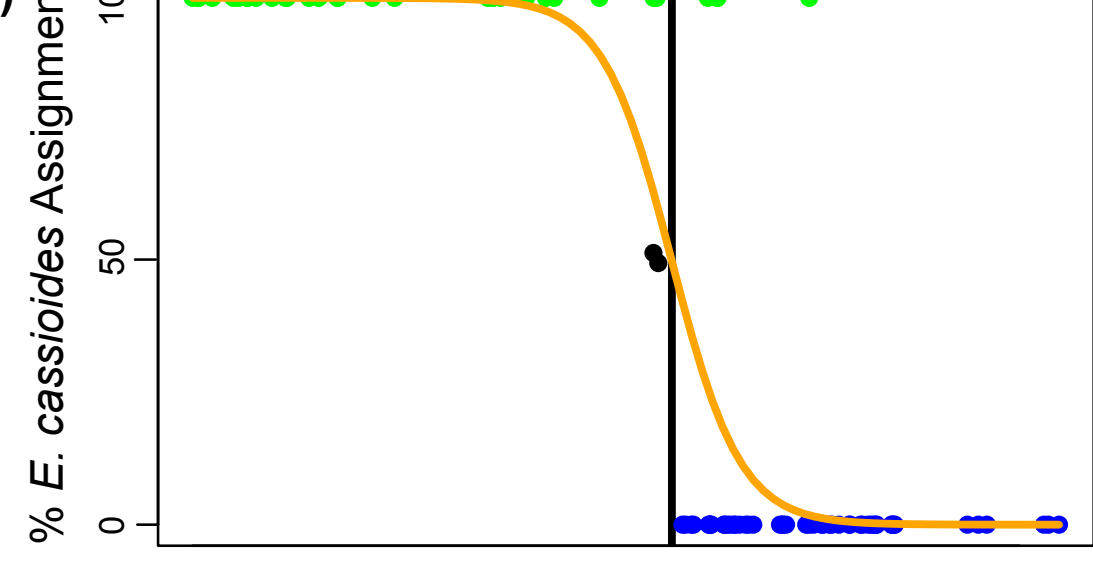

b)

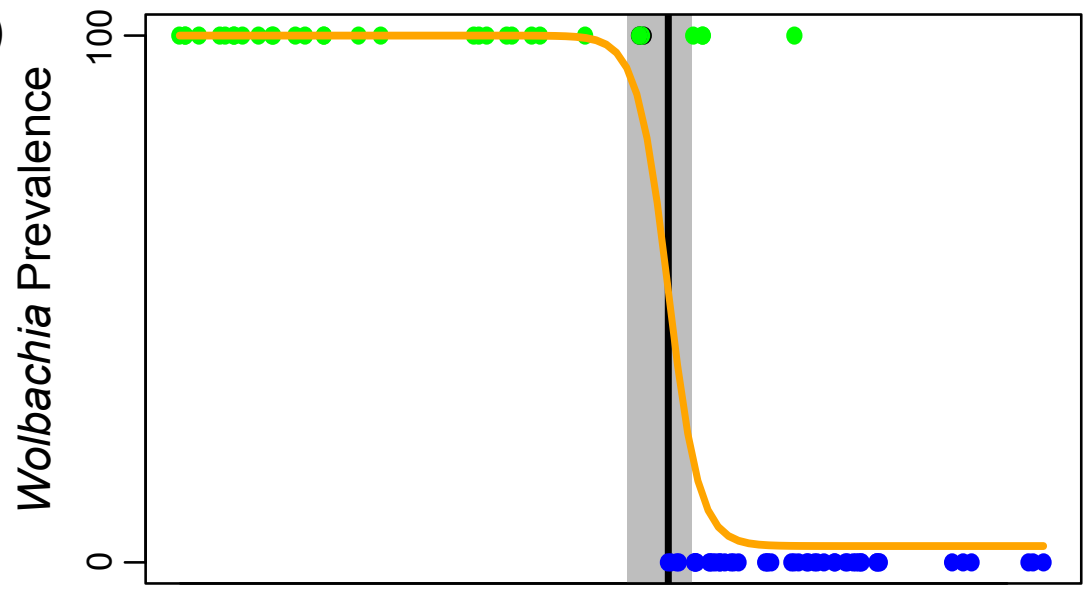

c)

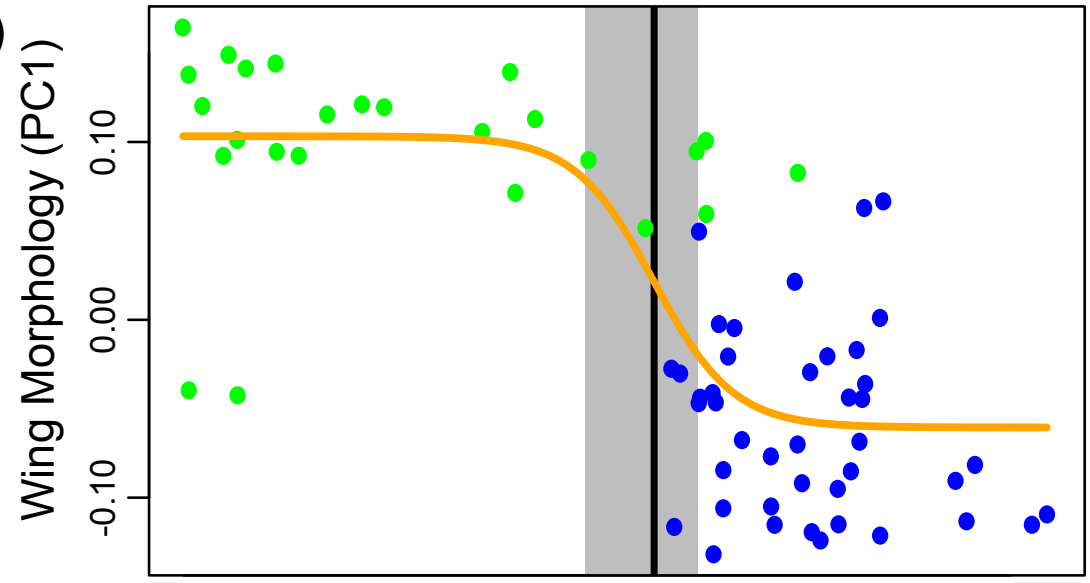

d)

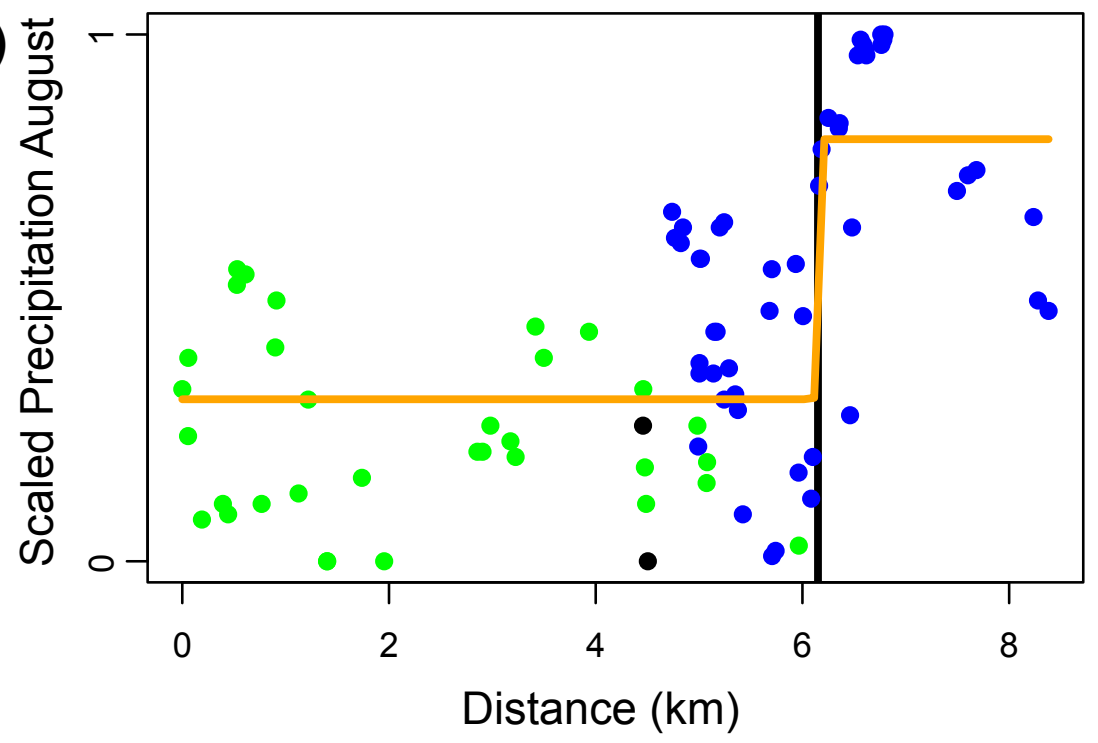

